\title{
Synchronization of time invariant uncertain delayed neural networks in finite time via improved sliding mode control
}

\author{
Jayanthi N. ${ }^{1}$, Santhakumari R. ${ }^{1,2}$ \\ ${ }^{1}$ Government Arts College, Coimbatore, India \\ ${ }^{2}$ Sri Ramakrishna College of Arts and Science, Coimbatore, India
}

(Received 24 February 2021; Accepted 11 April 2021)

\begin{abstract}
This paper explores the finite-time synchronization problem of delayed complex valued neural networks with time invariant uncertainty through improved integral sliding mode control. Firstly, the master-slave complex valued neural networks are transformed into two real valued neural networks through the method of separating the complex valued neural networks into real and imaginary parts. Also, the interval uncertainty terms of delayed complex valued neural networks are converted into the real uncertainty terms. Secondly, a new integral sliding mode surface is designed by employing the master-slave concept and the synchronization error of master-slave systems such that the error system can converge to zero in finite-time along the constructed integral sliding mode surface. Next, a suitable sliding mode control is designed by using Lyapunov stability theory such that state trajectories of the system can be driven onto the pre-set sliding mode surface in finite-time. Finally, a numerical example is presented to illustrate the effectiveness of the theoretical results.
\end{abstract}

Keywords: sliding mode control, sliding mode surface, time-invariant uncertainty, time delay, neural networks.

2010 MSC: 37C $75,49 \mathrm{~J} 25,32 \mathrm{G} 34,93 \mathrm{C} 15,26 \mathrm{~A} 18$

DOI: $10.23939 / \mathrm{mmc} 2021.02 .228$

\section{Introduction}

Neural networks have always been very significant in recent years for researchers in different fields, such as parallel computation, pattern recognition in computer science, designing associative memories and solving optimization problems. It is well known that due to the finite switching speed of amplifiers, a time delay is likely to exist and occur in signal transmission between neurons in the electronic implementation of neural networks, which will affect the stability of neural networks. In addition to time-delayed features of such neural networks, there may also be some complexities, such as disruptions and component variations, which may lead to very complex dynamic behaviours such as oscillations, synchronization, bifurcation and chaos. Moreover, most applications depend heavily on the dynamical behaviours of recurrent neural networks (see [1-7]). As a result, for many decades, several researchers have focused their efforts on the study and synthesis problems of neural network dynamics.

It is worth noting that most of neural network applications involve complex signals, and hence the study of complex-valued neural networks is essential for many real-world devices [8-16]. For example, a single real-valued neuron cannot deal with the problems in the detection of symmetry and XOR problems, but a single complex-valued neuron with orthogonal decision boundaries can successfully deal with them. Therefore for both engineering and science, the analysis of complex-valued neural networks is very necessary and important. As the extension of real-valued neural networks, complex-valued neural networks have complex-valued states, complex-valued connection weights, and complex-valued activation functions. Due to these characteristics, complex-valued neural networks have more abundant properties than real-valued neural networks. Some important findings have been published in the rapid development of complex-valued neural networks. For instance, the fixed-time synchronization problem for complex-valued BAM neural networks with time delay is investigated in [8]. Based on the Lya- 
punov direct method and fixed-time stability theorems, a novel fixed time synchronization condition is given. In [9], finite-time cluster synchronization of fractional-order complex-valued neural networks with nonlinear coupling is studied by utilizing the complex non-decomposition method. Finite-time synchronization between two delayed diffusive complex-valued neural networks with discontinuous activations is discussed in [10].

Synchronization phenomena have drawn much attention from researchers since the first discovery of synchronization made by Huygens in the 17th century. Synchronization or consensus is now commonly accepted as a kind of collective motion action which is exhibited in many natural systems [17]. In essence, synchronization is a form of self-organization or emergent phenomenon. It has been demonstrated that many real-world problems have close relationships with network synchronization. Various synchronization notations have been extended for its potential applications in various fields, such as complete synchronization [18], generalized synchronization [19], phase synchronization [20], projective synchronization [21], lag synchronization [22], cluster synchronization [23], and adaptive synchronization [24]. To date, most of the existing synchronization results for dynamic networks are asymptotic synchronization, which means that the convergence rate is at best exponential with infinite timesetting. In other words, states cannot reach synchronization in the finite time. Obviously, finite-time synchronization work is more desirable. Finite-time synchronization means the optimality in convergence or settling time. Minimizing synchronization time is therefore necessary for the attainment of fast communication synchrony. Further research on the problems of finite/fixed time-synchronization of complex-valued neural networks can be found in [8-10].

In addition, many different control strategies have been developed among the above results, such as pinning control [4,24], impulsive control [12,14,21], adaptive control [23, 24], feedback control [7], sliding control [25], intermittent control and sliding mode control (SMC). It should be noted that SMC is an effective control method and the main feature of SMC is to force the system states from the initial states to some predefined sliding mode surface with the switched control legislation. As a result, the desired benefits such as robustness, ability to track and insensitivity to external disturbances can be achieved. SMC has recently been used in various non-linear systems and complex networks. Although the above neural network synchronizations are widely studied, the neurons in the neural network are considered to be real-value. However, complex-valued variables are more frequent. As far as we know, few works have been devoted to the problem of finite-time synchronization for complex valued neural networks, which provide space to challenge this problem.

In view of the above analysis, this paper is intended to examine synchronization of complex valued neural networks with time delays in finite time. This paper's novelty lies in the following aspects.

1) For the sake of efficient research on synchronization of a class of $n$-dimensional complex valued neural networks, respective complex-valued neural networks are transformed into $2 n$-dimensional real-valued neural networks.

2) The designed integral sliding mode controller is able to synchronize the slave system with the corresponding master system in finite time, and the results obtained are less conservative than the previous works.

3) Those theoretical findings may provide new ideas for complex valued nonlinear systems to solve some real world problems.

Notations: In this paper, $\mathbb{R}^{n}$ is represented as $n$-dimensional Euclidean space. The Euclidean norm and 1 norm vector is denoted by $\|\cdot\|$ and $\|\cdot\|_{1}$ respectively.

\section{Preliminaries}

In this section, few related definitions, lemmas and assumptions are given to find the finite-time synchronization of time-invariant uncertainty complex-valued neural network (UCVNNs) with time delay. 
Definition 1. The drive-response systems are said to be finite-time synchronized if there exists a positive constant $T$ such that it satisfies the condition $0<T<\infty$ and also it depends on initial values of drive response systems, for any solution $\left(p_{1}, p_{2}, \ldots, p_{n}, q_{1}, q_{2}, \ldots, q_{n}\right)^{T}$ of drive system and $\left(u_{1}, u_{2}, \ldots, u_{n}, v_{1}, v_{2}, \ldots, v_{n}\right)^{T}$ of response system, then we have

$$
\lim _{t \rightarrow t_{0}+T}\left|\left(p_{i}(t), q_{i}(t)\right)-\left(u_{i}(t), v_{i}(t)\right)\right|=0
$$

and

$$
\left|\left(p_{i}(t), q_{i}(t)\right)-\left(u_{i}(t), v_{i}(t)\right)\right|=0, \quad t_{0}+T \leqslant t,
$$

where the norm of $\mathbb{R}$ is denoted by $|\cdot|$.

According to Definition 1, in order to prove the drive response systems are finite time synchronized, it is enough to prove for any constant $T$

$$
\lim _{t \rightarrow t_{0}+T}\left|e_{i}(t)\right|=0, \quad\left|e_{i}(t)\right|=0, \quad T+t_{0} \leqslant t .
$$

Rest of this paper, we use the following assumptions:

Assumption 1. There exist non-negative constants $\theta_{q}^{R R}, \theta_{q}^{I R}, \theta_{q}^{R I}, \theta_{q}^{I I}, \gamma_{q}^{R R}, \gamma_{q}^{I R}, \gamma_{q}^{R I}, \gamma_{q}^{I I}$ such that $\forall p_{1}, q_{1}, p_{2}, q_{2} \in \mathbb{R}$, where $p, q=1,2, \ldots, n$

$$
\begin{gathered}
\left|f_{q}^{R}\left(p_{1}, q_{1}\right)-f_{q}^{R}\left(p_{2}, q_{2}\right)\right| \leqslant \theta_{q}^{R R}\left|p_{1}-p_{2}\right|+\theta_{q}^{R I}\left|q_{1}-q_{2}\right|, \\
\left|f_{q}^{I}\left(p_{1}, q_{1}\right)-f_{q}^{I}\left(p_{2}, q_{2}\right)\right| \leqslant \theta_{q}^{I R}\left|p_{1}-p_{2}\right|+\theta_{q}^{I I}\left|q_{1}-q_{2}\right|, \\
\left|g_{q}^{R}\left(p_{1}, q_{1}\right)-g_{q}^{R}\left(p_{2}, q_{2}\right)\right| \leqslant \gamma_{q}^{R R}\left|p_{1}-p_{2}\right|+\gamma_{q}^{R I}\left|q_{1}-q_{2}\right|, \\
\left|g_{q}^{I}\left(p_{1}, q_{1}\right)-g_{q}^{I}\left(p_{2}, q_{2}\right)\right| \leqslant \gamma_{q}^{I R}\left|p_{1}-p_{2}\right|+\gamma_{q}^{I I}\left|q_{1}-q_{2}\right| .
\end{gathered}
$$

Lemma 1. Assume that there is a Lyapunov function $V(t)$ determined in the vicinity $U \subset \mathbb{R}$ of the origin and it satisfies

$$
\dot{V}(t)+\phi V(t)+\chi V^{a}(t) \leqslant 0, \quad \forall t \in U /\{0\},
$$

where $\phi>0, \chi>0$ and $a \in(0,1)$. Therefore, the finite time stability of system origin holds and finite time $T$ meets

$$
T(t) \leqslant \frac{1}{\phi(1-a)} \ln \left(\frac{\phi V^{1-a}\left(t_{0}\right)+\chi}{\chi}\right),
$$

where $t_{0}$ is the initial value of $t$.

Remark 1. It should be remembered that very little attention has been paid to finite-time synchronization of interval time-invariant uncertain complex valued neural networks (UCVNNs) with time delay through integral sliding mode control. To the best of our knowledge, there is no works related to finite-time synchronization for time-invariant UCVNNs with time delay via integral sliding mode controller in the previous literatures, which motivates the work of this paper.

\subsection{Problem description}

In this paper, we consider the following class of time-invariant UCVNNs with time delay as:

$$
\dot{z}(t)=-D(t) z(t)+A(t) f(z(t))+B(t) g z(t-\tau)+I,
$$

where $z(t) \in \mathbb{C}^{n}$ is the state vector; $D(t), A(t), B(t)$ are the $n \times n$ dimensional time-variant matrices; $f(\cdot), g(\cdot)$ are the activation functions without and with time delay respectively; the time delay $\tau$ satisfies $0<\tau$. The initial condition of system (1) is represented by $z(r)=\varrho(r), r \in\left[t_{0}-\tau, t_{0}\right]$, where $t_{0} \geqslant 0, \varrho(r)$ is complex function on $\left[t_{0}-\tau, t_{0}\right]$. Now we can separate (1) into its real and imaginary parts by using the properties of complex number as follows:

Mathematical Modeling and Computing, Vol. 8, No. 2, pp. 228-240 (2021) 


$$
\left\{\begin{aligned}
\dot{x}^{R}(t)= & -D(t) x^{R}(t)+A^{R}(t) f^{R}\left(x^{R}(t), y^{I}(t)\right)-A^{I}(t) f^{I}\left(x^{R}(t), y^{I}(t)\right) \\
& +B^{R}(t) g^{R}\left(x^{R}(t-\tau), y^{I}(t-\tau)\right)-B^{I}(t) g^{I}\left(x^{R}(t-\tau), y^{I}(t-\tau)\right)+I^{R}, \\
\dot{y}^{I}(t)= & -D(t) y^{I}(t)+A^{R}(t) f^{I}\left(x^{R}(t), y^{I}(t)\right)+A^{I}(t) f^{R}\left(x^{R}(t), y^{I}(t)\right) \\
& +B^{R}(t) g^{I}\left(x^{R}(t-\tau), y^{I}(t-\tau)\right)+B^{I}(t) g^{R}\left(x^{R}(t-\tau), y^{I}(t-\tau)\right)+I^{I},
\end{aligned}\right.
$$

where $x^{R}(t), y^{I}(t)$ are the real and imaginary parts of $z(t) ; A^{R}(t), A^{I}(t), B^{R}(t), B^{I}(t), I^{R}, I^{I}, f^{R}(\cdot, \cdot)$, $f^{I}(\cdot, \cdot), g^{R}(\cdot, \cdot), g^{I}(\cdot, \cdot)$ are the real and imaginary parts of $A(t), B(t), I, f(\cdot), g(\cdot)$ respectively. The time-variant matrices $D(t), A^{R}(t), B^{R}(t), A^{I}(t), B^{I}(t)$ are assumed as follows:

$$
\begin{aligned}
& D(t) \in D=\left[D^{-}, D^{+}\right]=\left\{\left(d_{i j}(t)\right)_{n \times n}: d_{i j}^{-} \leqslant d_{i j}(t) \leqslant d_{i j}^{+}, 1 \leqslant i, j \leqslant n, t \in \mathbb{R}\right\}, \\
& A^{R}(t) \in A^{R}=\left[A^{R-}, A^{R+}\right]=\left\{\left(a_{i j}^{R}(t)\right)_{n \times n}: a_{i j}^{R-}(t) \leqslant a_{i j}^{R}(t) \leqslant a_{i j}^{R+}(t), t \in \mathbb{R}\right\}, \\
& A^{R}(t) \in B^{R}=\left[B^{R-}, B^{R+}\right]=\left\{\left(b_{i j}^{R}(t)\right)_{n \times n}: b_{i j}^{R-}(t) \leqslant b_{i j}^{R}(t) \leqslant b_{i j}^{R+}(t), t \in \mathbb{R}\right\}, \\
& A^{I}(t) \in A^{I}=\left[A^{I-}, A^{I+}\right]=\left\{\left(a_{i j}^{I}(t)\right)_{n \times n}: a_{i j}^{I-}(t) \leqslant a_{i j}^{I}(t) \leqslant a_{i j}^{I+}(t), t \in \mathbb{R}\right\}, \\
& B^{I}(t) \in B^{I}=\left[B^{I-}, B^{I+}\right]=\left\{\left(b_{i j}^{I}(t)\right)_{n \times n}: b_{i j}^{I-}(t) \leqslant b_{i j}^{I}(t) \leqslant b_{i j}^{I+}(t), t \in \mathbb{R}\right\},
\end{aligned}
$$

where,

$$
\begin{aligned}
& D^{-}=\left(d_{i j}^{-}\right)_{n \times n}, \quad A^{R-}=\left(a_{i j}^{R-}\right)_{n \times n}, \quad B^{R-}=\left(b_{i j}^{R-}\right)_{n \times n}, \quad A^{I-}=\left(a_{i j}^{I-}\right)_{n \times n}, \quad B^{I-}=\left(b_{i j}^{I-}\right)_{n \times n}, \\
& D^{+}=\left(d_{i j}^{+}\right)_{n \times n}, \quad A^{R+}=\left(a_{i j}^{R+}\right)_{n \times n}, \quad B^{R+}=\left(b_{i j}^{R+}\right)_{n \times n}, \quad A^{I+}=\left(a_{i j}^{I+}\right)_{n \times n}, \quad B^{I+}=\left(b_{i j}^{I+}\right)_{n \times n} \text {. }
\end{aligned}
$$

In order to study the finite-time synchronization behavior of interval time invariant UCVNNs the following discussion is needed, therefore the time-variant matrices $D(t), A^{R}(t), A^{I}(t), B^{R}(t), B^{I}(t)$ can be described as:

$$
\begin{aligned}
D(t) & =\bar{D}+\Delta D(t), & & \\
A^{R}(t) & =\bar{A}^{R}+\Delta A^{R}(t), & A^{I}(t) & =\bar{A}^{I}+\Delta A^{I}(t), \\
B^{R}(t) & =\bar{B}^{R}+\Delta B^{R}(t), & B^{I}(t) & =\bar{B}^{I}+\Delta B^{I}(t),
\end{aligned}
$$

where,

$$
\begin{array}{rlrl}
\bar{D} & =\frac{D^{-}+D^{+}}{2}=\left(\frac{d_{i j}^{-}+d_{i j}^{+}}{2}\right)_{n \times n}, & \Delta D(t) & =\sum_{i, j=1}^{n} e_{i} \frac{c_{i j}^{(1)} E_{i j}^{(1)}(t) \tilde{d}_{i j}}{c_{i j}^{(1)}-J_{i j}^{(1)} E_{i j}^{(1)}(t)} e_{j}^{T}, \\
\bar{A}^{R}=\frac{A^{R-}+A^{R+}}{2}=\left(\frac{a_{i j}^{R-}+a_{i j}^{R+}}{2}\right)_{n \times n}, & \Delta A^{R}(t)=\sum_{i, j=1}^{n} e_{i} \frac{c_{i j}^{(2)} E_{i j}^{(2)}(t) \tilde{a}_{i j}^{R}}{c_{i j}^{(2)}-J_{i j}^{(2)} E_{i j}^{(2)}(t)} e_{j}^{T}, \\
\bar{B}^{R}=\frac{B^{R-}+B^{R+}}{2}=\left(\frac{b_{i j}^{R-}+b_{i j}^{R+}}{2}\right)_{n \times n}, & \Delta B^{R}(t)=\sum_{i, j=1}^{n} e_{i} \frac{c_{i j}^{(3)} E_{i j}^{(3)}(t) \tilde{b}_{i j}^{R}}{c_{i j}^{(3)}-J_{i j}^{(3)} E_{i j}^{(3)}(t)} e_{j}^{T}, \\
\bar{A}^{I}=\frac{A^{I-}+A^{I+}}{2}=\left(\frac{a_{i j}^{I-}+a_{i j}^{I+}}{2}\right)_{n \times n}, & \Delta A^{I}(t)=\sum_{i, j=1}^{n} e_{i} \frac{c_{i j}^{(4)} E_{i j}^{(4)}(t) \tilde{a}_{i j}^{I}}{c_{i j}^{(4)}-J_{i j}^{(4)} E_{i j}^{(4)}(t)} e_{j}^{T}, \\
\bar{B}^{I}=\frac{B^{I-}+B^{I+}}{2}=\left(\frac{b_{i j}^{I-}+b_{i j}^{I+}}{2}\right)_{n \times n}, & \Delta B^{I}(t)=\sum_{i, j=1}^{n} e_{i} \frac{c_{i j}^{(5)} E_{i j}^{(5)}(t) \tilde{b}_{i j}^{I}}{c_{i j}^{(5)}-J_{i j}^{(5)} E_{i j}^{(5)}(t)} e_{j}^{T} .
\end{array}
$$

Here, $E_{i j}^{(k)}(t)(k=1,2,3,4,5)$ are unknown constants satisfying

$$
\frac{c_{i j}^{(k)}}{J_{i j}^{(k)}-c_{i j}^{(k)}} \leqslant E_{i j}^{(k)}(t) \leqslant \frac{c_{i j}^{(k)}}{J_{i j}^{(k)}+c_{i j}^{(k)}}
$$

where, $\left|J_{i j}^{(k)}\right|<c_{i j}^{(k)}, 0<c_{i j}^{(k)} \in \mathbb{R}, \tilde{d}_{i j}, \tilde{a}_{i j}^{R}, \tilde{a}_{i j}^{I}, \tilde{b}_{i j}^{R}, \tilde{b}_{i j}^{I}$ are constants which satisfy 


$$
\tilde{d}_{i j}=\frac{d_{i j}^{+}-d_{i j}^{-}}{2}, \quad \tilde{a}_{i j}^{R}=\frac{a_{i j}^{R+}-a_{i j}^{R-}}{2}, \quad \tilde{a}_{i j}^{I}=\frac{a_{i j}^{I+}-a_{i j}^{I-}}{2}, \quad \tilde{b}_{i j}^{R}=\frac{b_{i j}^{R+}-b_{i j}^{R-}}{2}, \quad \tilde{b}_{i j}^{I}=\frac{b_{i j}^{I+}-b_{i j}^{I-}}{2} .
$$

The time-varient uncertain matrices $\Delta D(t), \Delta A^{R}(t), \Delta A^{I}(t), \Delta B^{R}(t)$ and $\Delta B^{I}(t)$ can be described in the following vector form:

$$
\left[\Delta D(t) \Delta A^{R}(t) \Delta A^{I}(t) \Delta B^{R}(t) \Delta B^{I}(t)\right]=\mathcal{M C} E(t)(\mathcal{C}-J E(t))^{-1} N,
$$

where $\mathcal{M}=[M M M M M], N=\operatorname{diag}\left\{N_{1}, N_{2}, N_{3}, N_{4}, N_{5}\right\}, J=\operatorname{diag}\left\{J_{1}, J_{2}, J_{3}, J_{4}, J_{5}\right\}$ and $C=\operatorname{diag}\left\{C_{1}, C_{2}, C_{3}, C_{4}, C_{5}\right\}$ with

$$
\begin{aligned}
M & =\left[M_{1}, M_{2}, \ldots, M_{n}\right], & N_{i} & =\left[N_{i 1}^{T}, N_{i 2}^{T}, \ldots, N_{i n}^{T}\right], \\
J_{k} & =\operatorname{diag}\left\{J_{1}^{(k)}, J_{2}^{(k)}, \ldots, J_{n}^{(k)}\right\}, & C_{k} & =\operatorname{diag}\left\{C_{1}^{(k)}, C_{2}^{(k)}, \ldots, C_{n}^{(k)}\right\}, \\
M_{i} & =\left[e_{1}, e_{2}, \ldots, e_{n}\right], & N_{1 j} & =\left[\tilde{d}_{j 1} e_{1}, \tilde{d}_{j 2} e_{2}, \ldots, \tilde{d}_{j n} e_{n}\right], \\
N_{2 j} & =\left[\tilde{a}_{j 1}^{R} e_{1}, \tilde{a}_{j 2}^{R} e_{2}, \ldots, \tilde{a}_{j n}^{R} e_{n}\right], & N_{3 j} & =\left[\tilde{a}_{j 1}^{I} e_{1}, \tilde{a}_{j 2}^{I} e_{2}, \ldots, \tilde{a}_{j n}^{I} e_{n}\right], \\
N_{4 j} & =\left[\tilde{b}_{j 1}^{R} e_{1}, \tilde{b}_{j 2}^{R} e_{2}, \ldots, \tilde{b}_{j n}^{R} e_{n}\right], & N_{5 j} & =\left[\tilde{b}_{j 1}^{I} e_{1}, \tilde{b}_{j 2}^{I} e_{2}, \ldots, \tilde{b}_{j n}^{I} e_{n}\right], \\
J_{j}^{(k)} & =\operatorname{diag}\left\{J_{j 1}^{(k)}, J_{j 2}^{(k)}, \ldots, J_{j n}^{(k)}\right\}, & C_{j}^{(k)} & =\operatorname{diag}\left\{C_{j 1}^{(k)}, C_{j 2}^{(k)}, \ldots, C_{j n}^{(k)}\right\}
\end{aligned}
$$

and $E(t)=\operatorname{diag}\left\{E_{1}(t), E_{2}(t), E_{3}(t), E_{4}(t), E_{5}(t)\right\}$ is an unknown time-varying matrix and it is denoted by

$$
\begin{gathered}
E_{k}(t)=\operatorname{diag}\left\{E_{1}^{(k)}(t), E_{2}^{(k)}(t), \ldots, E_{n}^{(k)}(t)\right\}, \quad E_{j}^{(k)}(t)=\operatorname{diag}\left\{E_{j 1}^{(k)}(t), E_{j 2}^{(k)}(t), \ldots, E_{j n}^{(k)}(t)\right\}, \\
j=1,2, \ldots, n .
\end{gathered}
$$

Then the uncertainty matrix is denoted as:

$$
\begin{gathered}
{\left[\Delta D(t) \Delta A^{R}(t) \Delta A^{I}(t) \Delta B^{R}(t) \Delta B^{I}(t)\right]=\mathcal{M} \Delta(t) N} \\
\Delta(t)=\operatorname{diag}\left\{\Delta_{1}(t), \Delta_{2}(t), \Delta_{3}(t), \Delta_{4}(t), \Delta_{5}(t)\right\}=C E(t)(C-J E(t))^{-1} .
\end{gathered}
$$

Then we can rewrite the system (2) in the following form:

$$
\left\{\begin{aligned}
\dot{x}^{R}(t)= & -(\bar{D}+\Delta D(t)) x^{R}(t)+\left(\bar{A}^{R}+\Delta A^{R}(t)\right)\left(f^{R}\left(x^{R}(t), y^{I}(t)\right)\right) \\
& -\left(A^{I-}+\Delta A^{I}(t)\right)\left(f^{I}\left(x^{R}(t), x^{I}(t)\right)\right)+\left(\bar{B}^{R}+\Delta B^{R}(t)\right)\left(g^{R}\left(x^{R}(t-\tau), y^{I}(t-\tau)\right)\right) \\
& -\left(B^{I-}+\Delta B^{I}(t)\right)\left(g^{I}\left(x^{R}(t-\tau), x^{I}(t-\tau)\right)\right)+I^{R} \\
\dot{y}^{I}(t)= & -(\bar{D}+\Delta D(t)) y^{I}(t)+\left(\bar{A}^{R}+\Delta A^{R}(t)\right)\left(f^{I}\left(x^{R}(t), y^{I}(t)\right)\right) \\
& +\left(A^{I-}+\Delta A^{I}(t)\right)\left(f^{R}\left(x^{R}(t), x^{I}(t)\right)\right)+\left(\bar{B}^{R}+\Delta B^{R}(t)\right)\left(g^{I}\left(x^{R}(t-\tau), y^{I}(t-\tau)\right)\right) \\
& +\left(B^{I-}+\Delta B^{I}(t)\right)\left(g^{R}\left(x^{R}(t-\tau), x^{I}(t-\tau)\right)\right)+I^{I} .
\end{aligned}\right.
$$

If we refer to model (5), as the master system and the corresponding slave system are the following ones denoted by:

$$
\left\{\begin{aligned}
\dot{\hat{x}}^{R}(t)= & -\left(\bar{D}_{1}+\Delta D_{1}(t)\right) \hat{x}^{R}(t)+\left(\bar{A}_{1}^{R}+\Delta A_{1}^{R}(t)\right)\left(\bar{f}^{R}\left(\hat{x}^{R}(t), \hat{y}^{I}(t)\right)\right) \\
& -\left(\bar{A}_{1}^{I}+\Delta A_{1}^{I}(t)\right)\left(\bar{f}^{I}\left(\hat{x}^{R}(t), \hat{y}^{I}(t)\right)\right)+\left(\bar{B}_{1}^{R}+\Delta B_{1}^{R}(t)\right)\left(\bar{g}^{R}\left(\hat{x}^{R}(t-\tau), \hat{y}^{I}(t-\tau)\right)\right) \\
& -\left(\bar{B}_{1}^{I}+\Delta B_{1}^{I}(t)\right)\left(\bar{g}^{I}\left(\hat{x}^{R}(t-\tau), \hat{y}^{I}(t-\tau)\right)\right)+I^{R}+u^{R}(t), \\
\dot{\hat{y}}^{I}(t)= & -\left(\bar{D}_{1}+\Delta D_{1}(t)\right) \hat{y}^{I}(t)+\left(\bar{A}_{1}^{R}+\Delta A_{1}^{R}(t)\right)\left(\bar{f}^{I}\left(\hat{x}^{R}(t), \hat{y}^{I}(t)\right)\right) \\
& +\left(\bar{A}_{1}^{I}+\Delta A_{1}^{I}(t)\right)\left(\bar{f}^{R}\left(\hat{x}^{R}(t), \hat{y}^{I}(t)\right)\right)+\left(\bar{B}_{1}^{R}+\Delta B_{1}^{R}(t)\right)\left(\bar{g}^{I}\left(\hat{x}^{R}(t-\tau), \hat{y}^{I}(t-\tau)\right)\right) \\
& +\left(\bar{B}_{1}^{I}+\Delta B_{1}^{I}(t)\right)\left(\bar{g}^{R}\left(\hat{x}^{R}(t-\tau), \hat{y}^{I}(t-\tau)\right)\right)+I^{I}+u^{I}(t),
\end{aligned}\right.
$$

where, $u^{R}(t), u^{I}(t)$ are control inputs and the remaining terms are defined in the same way as in the drive system (2). 
Remark 2. We can know that the system (5) is the real-valued neural networks that correspond to the complex-valued system (2), according to the above analysis. The problem of finite-synchronization of the system (2), is then transformed into the problem of finite-time synchronization of the real-valued system (5). Whereas the time-invariant uncertain neural network (5) and (6) can be asynchronous, when some of the network's parameters or time delays are chosen appropriately. Now we will investigate the finite-time synchronization conditions of the time-invariant uncertain real-valued neural networks (5) and (6) for the corresponding time-invariant uncertain complex-valued neural networks.

Let

$$
\begin{aligned}
e^{R}(t) & =\dot{x}^{R}(t)-\dot{\hat{x}}^{R}(t), \quad e^{I}(t)=\dot{y}^{I}(t)-\dot{\hat{y}}^{I}(t), \\
\phi^{R}\left(e^{R}(t), e^{I}(t)\right) & =f^{R}\left(x^{R}(t), y^{I}(t)\right)-f^{R}\left(\hat{x}^{R}(t), \hat{y}^{I}(t)\right), \\
\phi^{I}\left(e^{R}(t), e^{I}(t)\right) & =f^{I}\left(x^{R}(t), y^{I}(t)\right)-f^{I}\left(\hat{x}^{R}(t), \hat{y}^{I}(t)\right), \\
\xi^{R}\left(e^{R}(t-\tau), e^{I}(t-\tau)\right) & =g^{R}\left(x^{R}(t-\tau), y^{I}(t-\tau)\right)-g^{R}\left(\hat{x}^{R}(t-\tau), \hat{y}^{I}(t-\tau)\right), \\
\xi^{I}\left(e^{R}(t-\tau), e^{I}(t-\tau)\right) & =g^{I}\left(x^{R}(t-\tau), y^{I}(t-\tau)\right)-g^{I}\left(\hat{x}^{R}(t-\tau), \hat{y}^{I}(t-\tau)\right) .
\end{aligned}
$$

Now we can derive the error system of (5) and (6) and described them as follows:

$$
\begin{aligned}
\dot{e}^{R}(t)= & (\bar{D}+\Delta D(t)) e^{R}(t)+\left(\bar{A}^{R}+\Delta A^{R}(t)\right)\left(\phi^{R}\left(e^{R}(t), e^{I}(t)\right)\right)-\left(\bar{A}^{I}+\Delta A^{I}(t)\right)\left(\phi^{I}\left(e^{R}(t), e^{I}(t)\right)\right) \\
& +\left(\bar{B}^{R}+\Delta B^{R}(t)\right)\left(\xi^{R}\left(e^{R}(t-\tau), e^{I}(t-\tau)\right)\right)-\left(\bar{B}^{I}+\Delta B^{I}(t)\right)\left(\xi^{I}\left(e^{R}(t-\tau), e^{I}(t-\tau)\right)\right) \\
& +\left(\bar{D}_{1}-\bar{D}+\Delta D_{1}(t)-\Delta D(t)\right) \hat{x}^{R}(t)-\left(\bar{A}_{1}^{R}+\Delta A_{1}^{R}(t)\right)\left(f_{1}^{R}\left(\hat{x}^{R}(t), \hat{y}^{I}(t)\right)\right) \\
& +\left(\bar{A}^{R}+\Delta A^{R}(t)\right)\left(f^{R}\left(\hat{x}^{R}(t), \hat{y}^{I}(t)\right)\right)-\left(\bar{A}^{I}+\Delta A^{I}(t)\right)\left(f^{I}\left(\hat{x}^{R}(t), \hat{y}^{I}(t)\right)\right) \\
& +\left(\bar{A}_{1}^{I}+\Delta A_{1}^{I}(t)\right)\left(f_{1}^{I}\left(\hat{x}^{R}(t), \hat{y}^{I}(t)\right)\right)-\left(\bar{B}_{1}^{R}+\Delta B_{1}^{R}(t)\right)\left(g_{1}^{R}\left(\hat{x}^{R}(t-\tau), \hat{y}^{I}(t-\tau)\right)\right) \\
& +\left(\bar{B}^{R}+\Delta B^{R}(t)\right)\left(g^{R}\left(\hat{x}^{R}(t-\tau), \hat{y}^{I}(t-\tau)\right)\right)-\left(\bar{B}^{I}+\Delta B^{I}(t)\right)\left(g^{I}\left(\hat{x}^{R}(t-\tau), \hat{y}^{I}(t-\tau)\right)\right) \\
& +\left(\bar{B}_{1}^{I}+\Delta B_{1}^{I}(t)\right)\left(f_{1}^{I}\left(\hat{x}^{R}(t-\tau), \hat{y}^{I}(t-\tau)\right)\right)+I^{R}-I_{1}^{R}-u^{R}(t), \\
\dot{e}^{I}(t)= & -(\bar{D}+\Delta D(t)) e^{I}(t)+\left(\bar{A}^{R}+\Delta A^{R}(t)\right)\left(\phi^{I}\left(e^{R}(t), e^{I}(t)\right)\right)+\left(\bar{A}^{I}+\Delta A^{I}(t)\right)\left(\phi^{R}\left(e^{R}(t), e^{I}(t)\right)\right) \\
& +\left(\bar{B}^{R}+\Delta B^{R}(t)\right)\left(\xi^{I}\left(e^{R}(t-\tau), e^{I}(t-\tau)\right)\right)+\left(\bar{B}^{I}+\Delta B^{I}(t)\right)\left(\xi^{R}\left(e^{R}(t-\tau), e^{I}(t-\tau)\right)\right) \\
& +\left(\bar{D}_{1}-D+\Delta D_{1}(t)-\Delta D(t)\right) \hat{y}^{I}(t)+\left(\bar{A}^{R}+\Delta A^{R}(t)\right)\left(f^{I}\left(\hat{x}^{R}(t), \hat{y}^{I}(t)\right)\right) \\
& -\left(\bar{A}_{1}^{R}+\Delta A_{1}^{R}(t)\right)\left(f_{1}^{I}\left(\hat{x}^{R}(t), \hat{y}^{I}(t)\right)\right)+\left(\bar{A}^{I}+\Delta A^{I}(t)\right)\left(f^{R}\left(\hat{x}^{R}(t), \hat{y}^{I}(t)\right)\right) \\
& -\left(\bar{A}_{1}^{I}+\Delta A_{1}^{I}(t)\right)\left(f_{1}^{R}\left(\hat{x}^{R}(t), \hat{y}^{I}(t)\right)\right)+\left(\bar{B}^{R}+\Delta B^{R}(t)\right)\left(g^{I}\left(\hat{x}^{R}(t-\tau), \hat{y}^{I}(t-\tau)\right)\right) \\
& -\left(\bar{B}_{1}^{R}+\Delta B_{1}^{R}(t)\right)\left(g_{1}^{I}\left(\hat{x}^{R}(t-\tau), \hat{y}^{I}(t-\tau)\right)\right)+\left(\bar{B}^{I}+\Delta B^{I}(t)\right)\left(g^{R}\left(\hat{x}^{R}(t-\tau), \hat{y}^{I}(t-\tau)\right)\right) \\
& -\left(\bar{B}_{1}^{I}+\Delta B_{1}^{I}(t)\right)\left(g_{1}^{R}\left(\hat{x}^{R}(t-\tau), \hat{y}^{I}(t-\tau)\right)\right)-u^{I}(t)+I^{I}-I_{1}^{I} .
\end{aligned}
$$

Assumption 2. From (7) and (8) the continuous activation functions $f^{R}(\cdot, \cdot), f^{I}(\cdot, \cdot), \phi^{R}(\cdot, \cdot)$, $\phi^{I}(\cdot, \cdot), \quad g^{R}(\cdot, \cdot), g^{I}(\cdot, \cdot)$ satisfies $\left|f^{R}\left(\hat{x}^{R}(t), \quad \hat{y}^{I}(t)\right)\right| \leqslant \gamma_{1}^{R}\left|\hat{x}^{R}(t)\right|,\left|f^{I}\left(\hat{x}^{R}(t), \hat{y}^{I}(t)\right)\right| \leqslant \gamma_{1}^{I}\left|\hat{y}^{I}(t)\right|$, $\left|\phi^{R}\left(e^{R}(t), e^{I}(t)\right)\right| \leqslant \gamma_{1}^{R}\left|e^{R}(t)\right|,\left|\phi^{I}\left(e^{R}(t), e^{I}(t)\right)\right| \leqslant \gamma_{1}^{I}\left|e^{I}(t)\right|,\left|g^{R}\left(\hat{x}^{R}(t-\tau), \hat{y}^{I}(t-\tau)\right)\right| \leqslant \gamma_{2}^{R}\left|\hat{x}^{R}(t-\tau)\right|$, $\left|g^{I}\left(\hat{x}^{R}(t-\tau), \hat{y}^{I}(t-\tau)\right)\right| \leqslant \gamma_{2}^{I}\left|\hat{y}^{I}(t-\tau)\right|$. Then the following inequalities are holds:

$$
\begin{array}{cc}
\left\|f^{R}\left(\hat{x}^{R}(t), \hat{y}^{I}(t)\right)\right\| \leqslant\left\|\mathcal{L}_{1}^{R}\right\|\left|\hat{x}^{R}(t)\right|, & \left\|f^{R}\left(\hat{x}^{R}(t), \hat{y}^{I}(t)\right)\right\| \leqslant\left\|\mathcal{L}_{1}^{I}\right\|\left|\hat{y}^{I}(t)\right|, \\
\left\|\phi^{R}\left(e^{R}(t), e^{I}(t)\right)\right\| \leqslant\left\|\mathcal{L}_{1}^{R}\right\|\left|e^{R}(t)\right|, & \left\|\phi^{I}\left(e^{R}(t), e^{I}(t)\right)\right\| \leqslant\left\|\mathcal{L}_{1}^{I}\right\|\left|e^{I}(t)\right|, \\
\left\|g^{R}\left(\hat{x}^{R}(t-\tau), \hat{y}^{I}(t-\tau)\right)\right\| \leqslant\left\|\mathcal{L}_{2}^{R}\right\|\left|\hat{x}^{R}(t-\tau)\right|, \\
\left\|g^{R}\left(\hat{x}^{R}(t-\tau), \hat{y}^{I}(t-\tau)\right)\right\| \leqslant\left\|\mathcal{L}_{2}^{I}\right\|\left|\hat{y}^{I}(t-\tau)\right| .
\end{array}
$$

Assumption 3. Due to the limit of the error signals, we can get $\left\|e^{R}(t)\right\|_{1} \leqslant \zeta_{1}^{R},\left\|e^{R}(t)\right\| \leqslant \zeta_{2}^{R}$, $\left\|e^{I}(t)\right\|_{1} \leqslant \zeta_{1}^{I},\left\|e^{I}(t)\right\| \leqslant \zeta_{2}^{I}$, for any positive constant $\zeta_{1}^{R}, \zeta_{2}^{I}, \zeta_{1}^{I}, \zeta_{2}^{R}$. 
Remark 3. The main goal of this paper is to develop the sliding mode control (SMC) method and construct the suitable SMC for to achieve the master-slave finite-time synchronization of the complexvalued neural networks and it can be conveniently obtained in the next sections.

\section{SMC design}

In this section a new type of integral SMS (sliding mode surface) will be designed by using errors (7) and (8) which is different from [26,27]. In addition, using this integral SMS we can construct the integral SMC for the finite-time synchronization of the errors (7) and (8).

The novel integral SMS is firstly constructed as follows:

$$
\left\{\begin{array}{l}
S^{R}(t)=e^{R}(t)+\int_{0}^{t}\left[\omega_{1}^{R} e^{R}(r)+\zeta^{R} e^{\alpha_{1}^{R}}(r)\right] \operatorname{sign}(r) \mathrm{d} r \\
S^{I}(t)=e^{I}(t)+\int_{0}^{t}\left[\omega_{1}^{I} e^{I}(r)+\zeta^{I} e^{\alpha_{1}^{I}}(r)\right] \operatorname{sign}(r) \mathrm{d} r
\end{array}\right.
$$

and suitable sliding mode controller (SMC) is

$$
\left\{\begin{array}{l}
u^{R}(t)=\rho^{R}(t) \operatorname{sign}\left(s^{R}(t)\right)+\omega_{2}^{R} s^{R}(t)+\zeta_{2}^{R} s^{\alpha_{2}^{I}}(t) \\
u^{I}(t)=\rho^{I}(t) \operatorname{sign}\left(s^{I}(t)\right)+\omega_{2}^{I} s^{I}(t)+\zeta_{2}^{I} s^{\alpha_{2}^{R}}(t)
\end{array}\right.
$$

where,

$$
\begin{aligned}
\rho^{R}(t)= & {\left[\|\mathfrak{D}\|+\left(\left\|\mathfrak{A}^{R}\right\|+\left\|\mathfrak{B}^{R}\right\|\right) \delta^{R R}+\left(\left\|\mathfrak{A}^{I}\right\|+\left\|\mathfrak{B}^{I}\right\|\right) \delta^{I R}+\omega_{1}\right] \zeta_{2}^{R}+\left[\left(\left\|\mathfrak{A}^{R}\right\|+\left\|\mathfrak{B}^{R}\right\|\right) \delta^{R I}\right.} \\
& \left.+\left(\left\|\mathfrak{A}^{I}\right\|+\left\|\mathfrak{B}^{I}\right\|\right) \delta^{I I}\right] \zeta_{2}^{I}+\left[\|\mathfrak{D}\|+\left(\left\|\mathfrak{A}^{R}\right\|+\left\|\mathfrak{A}_{1}^{R}\right\|\right)\left\|\mathcal{L}_{1}^{R}\right\|\right]\left\|\hat{x}^{R}(t)\right\|+\left[\left\|\mathfrak{A}^{R}\right\|\left\|\mathcal{L}_{1}^{R}\right\|\right. \\
& \left.+\left\|\mathfrak{A}_{1}^{I}\right\|\left\|\mathcal{L}_{1}^{I}\right\|\right]\left\|\hat{y}^{I}(t)\right\|+\left[\left\|\mathfrak{B}^{R}\right\|\left\|\mathcal{L}_{2}^{R}\right\|+\left\|\mathfrak{B}_{1}^{I}\right\|\left\|\mathcal{L}_{2}^{I}\right\|\right]\left\|\hat{x}^{R}(t-\tau)\right\|+\left[\left\|\mathfrak{B}^{I}\right\|\left\|\mathcal{L}_{2}^{I}\right\|\right. \\
& \left.+\left\|\mathfrak{B}_{1}^{I}\right\|\left\|\mathcal{L}_{2}^{I}\right\|\right]\|\hat{y}(t-\tau)\|+\left(\zeta_{1} \varepsilon_{1}^{\alpha_{1}}\right)^{R}+\left\|I_{1}^{R}-\hat{I}_{1}^{R}\right\|, \\
\rho^{I}(t)= & {\left[\|\mathfrak{D}\|+\left(\left\|\mathfrak{A}^{R}\right\|+\left\|\mathfrak{B}^{R}\right\|\right) \delta^{R I}+\left(\left\|\mathfrak{A}^{I}\right\|+\left\|\mathfrak{B}^{I}\right\|\right) \delta^{R R}+\omega_{2}^{R}\right] \zeta_{2}^{R}+\left[\left(\left\|\mathfrak{A}^{R}\right\|+\left\|\mathfrak{B}^{R}\right\|\right) \delta^{I I}\right.} \\
& \left.+\left(\left\|\mathfrak{A}^{I}\right\|+\left\|\mathfrak{B}^{I}\right\|\right) \delta^{I R}+\omega_{2}^{I}\right] \zeta_{2}^{I}+\left[\left\|\mathfrak{D}^{*}\right\|+\left(\left\|\mathfrak{A}^{R}\right\|+\left\|\mathfrak{A}_{1}^{R}\right\|\right)\left\|\mathcal{L}_{1}^{I}\right\|\right]\left\|\hat{y}^{I}(t)\right\|+\left[\left\|\mathfrak{A}^{I}\right\|\left\|\mathcal{L}_{1}^{R}\right\|\right. \\
& \left.+\left\|\mathfrak{A}_{1}^{I}\right\|\left\|\mathcal{L}_{2}^{R}\right\|\right]\left\|\hat{x}^{R}(t)\right\|+\left[\left\|\mathfrak{B}^{R}\right\|\left\|\mathcal{L}_{1}^{I}\right\|+\left\|\mathfrak{B}_{1}^{R}\right\|\left\|\mathcal{L}_{2}^{I}\right\|\right]\left\|\hat{x}^{R}(t-\tau)\right\|+\left[\left\|\mathfrak{B}^{I}\right\|\left\|\mathcal{L}_{2}^{R}\right\|\right. \\
& \left.+\left\|\mathfrak{B}_{1}^{I}\right\|\left\|\mathcal{L}_{2}^{R}\right\|\right]\left\|\hat{y}^{I}(t-\tau)\right\| .
\end{aligned}
$$

Theorem 1. Suppose we take the integral SMS shown in (9) and the corresponding SMC shown in (10), then the finite-time synchronization can be guaranteed between the time-invariant uncertainty neural networks (5) and (6).

Proof. From equations (9) we have,

where,

$$
\begin{aligned}
\dot{S}(t) & =\dot{e}(t)+\vartheta_{1} \operatorname{sign}(t)-\vartheta_{2} \operatorname{sign}(0) \\
& = \begin{cases}\varrho \operatorname{sign}(0), & t>0, \\
\dot{e}(t), & t=0,\end{cases}
\end{aligned}
$$

$$
\begin{aligned}
& \dot{S}(t)=\left(\dot{S}^{R}(t), \dot{S}^{I}(t)\right)^{T}, \quad \dot{e}(t)=\left(\dot{e}^{R}(t), \dot{e}^{I}(t)\right)^{T}, \quad \vartheta_{1}=\left(\vartheta_{1}^{R}, \vartheta_{1}^{I}\right)^{T}, \quad \vartheta_{2}=\left(\vartheta_{2}^{R}, \vartheta_{2}^{I}\right)^{T}, \quad \varrho=\left(\varrho^{R}, \varrho^{I}\right), \\
& \vartheta_{1}^{R}=\left[\omega_{1} e^{R}(t)+\xi^{R} e_{1}^{\alpha_{1} R}(t)\right], \vartheta_{1}^{I}=\left[\omega_{1} e^{I}(t)+\xi^{I} e_{1}^{\alpha_{1} I}(t)\right], \\
& \left.\vartheta_{2}^{I}=\left[\omega_{1} e^{I}(0)+\xi^{I} e_{1}^{\alpha_{1} I}(0)\right], \varrho^{R}=\left[e^{R}(t)+\omega_{1}^{R} e^{R}(t)+\xi_{1}^{R} e^{\alpha_{1} R}(t)\right], \varrho^{I}=\left[\omega_{1} e^{R}(0)+\xi^{R} e_{1}^{\alpha_{1} R}(0)\right], \omega_{1}^{I} e^{I}(t)+\xi_{1}^{I} e^{\alpha_{1} I}(t)\right] .
\end{aligned}
$$

When we choose $t>0$

$\dot{S}^{R}(t)=-\mathfrak{D} e^{R}(t)+\mathfrak{A}^{R} e^{R}(x(t), y(t))-\mathfrak{A}^{I} \phi^{I}\left(e^{I}(x(t), y(t))\right)+\mathfrak{B}^{R} \psi^{R}\left(e^{R}(x(t-\tau), y(t-\tau))\right)$

Mathematical Modeling and Computing, Vol. 8, No. 2, pp. 228-240 (2021) 


$$
\begin{aligned}
& -\mathfrak{B}^{I} \psi^{I}\left(e^{I}(x(t-\tau), y(t-\tau))\right)+\mathfrak{D}^{*} \hat{x}(t)-\mathfrak{A}^{I} f^{I}(\hat{x}(t), \hat{y}(t))+\mathfrak{A}^{R} f^{R}(x(t), y(t)) \\
& -\mathfrak{A}^{I} f^{R}(x(t), y(t))+\mathfrak{A}_{1}^{I} f_{1}^{I}(x(t), y(t))+\mathfrak{B}^{R} g^{R}(\hat{x}(t-\tau), \hat{y}(t-\tau))-\mathfrak{B}_{1}^{R} g_{1}^{R}(\hat{x}(t-\tau), \hat{y}(t-\tau)) \\
& -\mathfrak{B}^{I} g^{I}(\hat{x}(t-\tau), \hat{y}(t-\tau))+\mathfrak{B}_{1}^{I} g_{1}^{I}(\hat{x}(t-\tau), \hat{y}(t-\tau))+I_{1}^{R}-\hat{I}_{1}^{R}+\omega_{1}^{R} e^{R}(t)+\xi_{1}^{R} e^{\alpha_{1}^{R}}(t) \\
& -\rho^{R}(t) \operatorname{sign}\left(S^{R}(t)\right)-\omega_{2}^{R} S^{R}(t)-\xi_{2}^{R} S^{\alpha_{2} R}(t), \\
\dot{S}^{I}(t)= & -\mathfrak{D} e^{I}(t)-\mathfrak{A}\left(\phi^{I}\left(e^{R}(t), e^{I}(t)\right)\right)+\mathfrak{A}^{I}\left(\phi^{R}\left(e^{R}(t), e^{I}(t)\right)\right)+\mathfrak{B}^{R}\left(\xi^{I}\left(e^{R}(t-\tau), e^{I}(t-\tau)\right)\right) \\
& +\mathfrak{B}^{I}\left(\xi^{R}\left(e^{R}(t-\tau), e^{I}(t-\tau)\right)\right)+\mathfrak{D}^{*} \hat{y}^{I}(t)+\mathfrak{A}^{R}\left(f^{I}\left(\hat{x}^{R}(t), \hat{y}^{I}(t)\right)\right)-\mathfrak{A}_{1}^{R}\left(f_{1}^{I}\left(\hat{x}^{R}(t), \hat{y}^{I}(t)\right)\right) \\
& +\mathfrak{A}^{I}\left(f^{R}\left(\hat{x}^{R}(t), \hat{y}^{I}(t)\right)\right)-\mathfrak{A}_{1}^{I}\left(f_{1}^{R}\left(\hat{x}^{R}(t), \hat{y}^{I}(t)\right)\right)+\mathfrak{B}^{R}\left(g^{I}\left(\hat{x}^{R}(t-\tau), \hat{y}^{I}(t-\tau)\right)\right) \\
& -\mathfrak{B}_{1}^{R}\left(g_{1}^{I}\left(\hat{x}^{R}(t-\tau), \hat{y}^{I}(t-\tau)\right)\right)+\mathfrak{B}^{I}\left(g^{R}\left(\hat{x}^{R}(t-\tau), \hat{y}^{I}(t-\tau)\right)\right) \\
& -\mathfrak{B}_{1}^{I}\left(g_{1}^{R}\left(\hat{x}^{R}(t-\tau), \hat{y}^{I}(t-\tau)\right)\right) \\
& -\hat{u}_{1}^{I}(t)+I^{I}-\hat{I}_{1}^{I}+\omega_{1}^{I} e^{I}(t)+\xi_{1}^{I} e^{\alpha_{1} I}(t)-\rho^{I}(t) \operatorname{sign}\left(S^{I}(t)\right)-\omega_{2}^{I} S^{I}(t)-\xi_{2}^{I} S^{\alpha_{2} I}(t),
\end{aligned}
$$

where, $\mathfrak{D}=\bar{D}+\Delta D(t), \mathfrak{A}^{R}=\bar{A}^{R}+\Delta A^{R}(t), \mathfrak{A}^{I}=\bar{A}^{I}+\Delta A^{I}(t), \mathfrak{B}^{R}=\bar{B}^{R}+\Delta B^{R}(t), \mathfrak{B}^{I}=\bar{B}^{I}+$ $\Delta B^{I}(t), \mathfrak{D}^{*}=\bar{D}_{1}-D+\Delta D_{1}(t)-\Delta D(t), \mathfrak{A}_{1}^{R}=\bar{A}_{1}^{R}+\Delta A_{1}^{R}(t), \mathfrak{A}_{1}^{I}=\bar{A}_{1}^{I}+\Delta A_{1}^{I}(t), \mathfrak{B}_{1}^{R}=\bar{B}_{1}^{R}+\Delta B_{1}^{R}(t)$, $\mathfrak{B}_{1}^{I}=\bar{B}_{1}^{I}+\Delta B_{1}^{I}(t)$.

Let us construct the following Lyapunov functional candidate

$$
V(t)=\frac{1}{2} S^{R^{T}}(t) S^{R}(t)+\frac{1}{2} S^{I^{T}}(t) S^{I}(t) .
$$

Taking the time derivative of (13) we can get

$$
\begin{aligned}
& \dot{V}(t)=S^{R^{T}}(t) \dot{S}^{R}(t)+S^{I^{T}}(t) \dot{S}^{I}(t) \\
& =S^{R^{T}}(t)\left[-\mathfrak{D} e^{R}(t)+\mathfrak{A}^{R} e^{R}(x(t), y(t))-\mathfrak{A}^{I} \phi^{I}\left(e^{I}(x(t), y(t))\right)+\mathfrak{B}^{R} \psi^{R}\left(e^{R}(x(t-\tau), y(t-\tau))\right)\right. \\
& -\mathfrak{B}^{I} \psi^{I}\left(e^{I}(x(t-\tau), y(t-\tau))\right)+\mathfrak{D}^{*} \hat{x}(t)-\mathfrak{A}^{I} f^{I}(\hat{x}(t), \hat{y}(t))+\mathfrak{A}^{R} f^{R}(x(t), y(t)) \\
& -\mathfrak{A}^{I} f^{R}(x(t), y(t))+\mathfrak{A}_{1}^{I} f_{1}^{I}(x(t), y(t))+\mathfrak{B}^{R} g^{R}(\hat{x}(t-\tau), \hat{y}(t-\tau))-\mathfrak{B}_{1}^{R} g_{1}^{R}(\hat{x}(t-\tau), \hat{y}(t-\tau)) \\
& -\mathfrak{B}^{I} g^{I}(\hat{x}(t-\tau), \hat{y}(t-\tau))+\mathfrak{B}_{1}^{I} g_{1}^{I}(\hat{x}(t-\tau), \hat{y}(t-\tau))+I_{1}^{R}-\hat{I}_{1}^{R}+\omega_{1}^{R} e^{R}(t)+\xi_{1}^{R} e^{\alpha_{1}^{R}}(t) \\
& \left.-\rho^{R}(t) \operatorname{sign}\left(S^{R}(t)\right)-\omega_{2} S(t)-\xi_{2}^{R} S^{\alpha_{2}}(t)\right]+S^{I^{T}}(t)\left[-\mathfrak{D} e^{I}(t)-\mathfrak{A}\left(\phi^{I}\left(e^{R}(t), e^{I}(t)\right)\right)\right. \\
& +\mathfrak{A}^{I}\left(\phi^{R}\left(e^{R}(t), e^{I}(t)\right)\right)+\mathfrak{B}^{R}\left(\xi^{I}\left(e^{R}(t-\tau), e^{I}(t-\tau)\right)\right)+\mathfrak{B}^{I}\left(\xi^{R}\left(e^{R}(t-\tau), e^{I}(t-\tau)\right)\right) \\
& +\mathfrak{D}^{*} \hat{y}^{I}(t)+\mathfrak{A}^{R}\left(f^{I}\left(\hat{x}^{R}(t), \hat{y}^{I}(t)\right)\right)-\mathfrak{A}_{1}^{R}\left(f_{1}^{I}\left(\hat{x}^{R}(t), \hat{y}^{I}(t)\right)\right)+\mathfrak{A}^{I}\left(f^{R}\left(\hat{x}^{R}(t), \hat{y}^{I}(t)\right)\right) \\
& -\mathfrak{A}_{1}^{I}\left(f_{1}^{R}\left(\hat{x}^{R}(t), \hat{y}^{I}(t)\right)\right)+\mathfrak{B}^{R}\left(g^{I}\left(\hat{x}^{R}(t-\tau), \hat{y}^{I}(t-\tau)\right)\right)-\mathfrak{B}_{1}^{R}\left(g_{1}^{I}\left(\hat{x}^{R}(t-\tau), \hat{y}^{I}(t-\tau)\right)\right) \\
& +\mathfrak{B}^{I}\left(g^{R}\left(\hat{x}^{R}(t-\tau), \hat{y}^{I}(t-\tau)\right)\right)-\mathfrak{B}_{1}^{I}\left(g_{1}^{R}\left(\hat{x}^{R}(t-\tau), \hat{y}^{I}(t-\tau)\right)\right)-\hat{u}_{1}^{I}(t)+I^{I}-\hat{I}_{1}^{I}+\omega_{1}^{I} e^{I}(t) \\
& \left.+\xi_{1}^{I} e^{\alpha_{1}^{I}}(t)-\rho^{I}(t) \operatorname{sign}\left(S^{I}(t)\right)-\omega_{2} S^{I}(t)-\xi_{2}^{I} S^{\alpha_{2}^{I}}(t)\right] \\
& =\left\|S^{R}(t)\right\|\left[\|\mathfrak{D}\|\left\|e^{R}(t)\right\|+\left\|\mathfrak{A}^{R}\right\| \phi^{R}\left(e^{R}(t), e^{I}(t)\right)\|+\| \mathfrak{A}^{I}\|\| \phi^{I}\left(e^{R}(t), e^{I}(t)\right) \|\right. \\
& +\left\|\mathfrak{B}^{R}\right\|\left\|\xi^{R}\left(e^{R}(t-\tau), e^{I}(t-\tau)\right)\right\|+\left\|\mathfrak{B}^{I}\right\|\left\|\xi^{I}\left(e^{R}(t-\tau), e^{I}(t-\tau)\right)\right\|+\left\|\mathfrak{D}^{*}\right\|\left\|\hat{x}^{R}\right\| \\
& +\left\|\mathfrak{A}^{I}\right\|\left\|f^{I}(\hat{x}(t), \hat{y}(t))\right\|+\left\|\mathfrak{A}^{R}\right\|\left\|f^{R}\left(\hat{x}^{R}(t), \hat{y}^{I}(t)\right)\right\|+\left\|\mathfrak{A}_{1}^{R}\right\|\left\|f_{1}^{R}\left(\hat{x}^{R}(t), \hat{y}^{I}(t)\right)\right\| \\
& +\left\|\mathfrak{A}_{1}^{I}\right\|\left\|f_{1}^{I}\left(x^{R}(t), y^{I}(t)\right)\right\|+\left\|\mathfrak{B}_{1}^{R}\right\|\left\|g_{1}^{R}\left(\hat{x}^{R}(t-\tau), \hat{y}^{I}(t-\tau)\right)\right\|+\left\|\mathfrak{B}_{1}^{I}\right\|\left\|g_{1}^{I}\left(\hat{x}^{R}(t-\tau), \hat{y}^{I}(t-\tau)\right)\right\| \\
& \left.+\left\|I^{R}-\hat{I}_{1}^{R}\right\|+\omega_{1}^{R}\left\|e^{R}(t)\right\|+\xi_{1}^{R}\left\|e^{\alpha_{1} R}(t)\right\|\right]-\rho^{R}(t)\left\|S^{R}(t)\right\|_{1}-\omega_{2} S^{R^{T}}(t) S^{R}(t) \\
& -\xi_{2}^{R} \sum_{i=1}^{n} S_{i}^{\left(\alpha_{2}+1\right)}(t)+\left\|S^{I}(t)\right\|\left[\|\mathfrak{D}\|\left\|e^{I}(t)\right\|+\left\|\mathfrak{A}^{R}\right\|\left\|\phi^{I}\left(e^{R}(t), e^{I}(t)\right)\right\|\right. \\
& +\left\|\mathfrak{A}^{I}\right\|\left\|\phi^{R}\left(e^{R}(t), e^{I}(t)\right)\right\|+\left\|\mathfrak{B}^{R}\right\|\left\|\xi^{I}\left(e^{R}(t-\tau), e^{I}(t-\tau)\right)\right\|+\left\|\mathfrak{B}^{I}\right\|\left\|\xi^{R}\left(e^{R}(t-\tau), e^{I}(t-\tau)\right)\right\| \\
& +\left\|\mathfrak{D}^{*}\right\|\left\|\hat{y}^{I}(t)\right\|+\left\|\mathfrak{A}^{R}\right\|\left\|f^{I}\left(\hat{x}^{R}(t), \hat{y}^{I}(t)\right)\right\|+\left\|\mathfrak{A}_{1}^{R}\right\|\left\|f_{1}^{I}\left(\hat{x}^{R}(t), \hat{y}^{I}(t)\right)\right\| \\
& +\left\|\mathfrak{A}^{I}\right\|\left\|f^{R}\left(\hat{x}^{R}(t), \hat{y}^{I}(t)\right)\right\|+\left\|\mathfrak{A}_{1}^{I}\right\|\left\|f_{1}^{R}\left(\hat{x}^{R}(t), \hat{y}^{I}(t)\right)\right\|+\left\|\mathfrak{B}^{R}\right\|\left\|g^{I}\left(\hat{x}^{R}(t-\tau), \hat{y}^{I}(t-\tau)\right)\right\|
\end{aligned}
$$




$$
\begin{aligned}
& +\left\|\mathfrak{B}_{1}^{R}\right\|\left\|g_{1}^{I}\left(\hat{x}^{R}(t-\tau), \hat{y}^{I}(t-\tau)\right)\right\|+\left\|\mathfrak{B}^{I}\right\|\left\|g^{R}\left(\hat{x}^{R}(t-\tau), \hat{y}^{I}(t-\tau)\right)\right\| \\
& \left.+\left\|\mathfrak{B}_{1}^{I}\right\|\left\|g_{1}^{R}\left(\hat{x}^{R}(t-\tau), \hat{y}^{I}(t-\tau)\right)\right\|+\left\|I^{I}-\hat{I}_{1}^{I}\right\|+\omega_{1}^{I}\left\|e^{I}(t)\right\|+\xi_{1}^{I}\left\|e^{\alpha_{1} I}(t)\right\|\right]-\rho^{I}(t)\left\|S^{I}(t)\right\|_{1} \\
& -\omega_{2} S^{I^{T}}(t) S^{I}(t)-\xi_{2}^{I} \sum_{i=1}^{n} S_{i}^{I\left(\alpha_{2}+1\right)}(t) .
\end{aligned}
$$

Then we set the inequalities $\left\|S^{R}(t)\right\| \leqslant\left\|S^{R}(t)\right\|_{1},\left\|S^{I}(t)\right\| \leqslant\left\|S^{I}(t)\right\|_{1}$ and $\left\|e^{\alpha_{1} R}(t)\right\| \leqslant$ $\left\|e^{\alpha_{1} R}(t)\right\|_{\left(1 / \alpha_{1}\right)}=\left[\left\|e^{R}(t)\right\|_{1}\right]^{\alpha_{1}},\left\|e^{\alpha_{1} I}(t)\right\| \leqslant\left\|e^{\alpha_{1} I}(t)\right\|_{\left(1 / \alpha_{1}\right)}=\left[\left\|e^{I}(t)\right\|_{1}\right]^{\alpha_{1}},\left(1 / 2 \leqslant \alpha_{1}<1\right),\left\|e^{R}(t)\right\| \leqslant$ $\xi_{2}^{R},\left\|e^{I}(t)\right\| \leqslant \xi_{2}^{I},\left\|e^{R}(\cdot)\right\| \leqslant \xi_{1},\left\|e^{I}(\cdot)\right\| \leqslant \xi_{2}$,

$$
\begin{aligned}
& \dot{V}(t) \leqslant\left\|S^{R}(t)\right\|_{1}\left[\|\mathfrak{D}\|\left\|e^{R}(t)\right\|+\left\|\mathfrak{A}^{R}\right\| \phi^{R}\left(e^{R}(t), e^{I}(t)\right)\|+\| \mathfrak{A}^{I}\|\| \phi^{I}\left(e^{R}(t), e^{I}(t)\right) \|\right. \\
& +\left\|\mathfrak{B}^{R}\right\|\left\|\xi^{R}\left(e^{R}(t-\tau), e^{I}(t-\tau)\right)\right\|+\left\|\mathfrak{B}^{I}\right\|\left\|\xi^{I}\left(e^{R}(t-\tau), e^{I}(t-\tau)\right)\right\|+\left\|\mathfrak{D}^{*}\right\|\left\|\hat{x}^{R}\right\| \\
& +\left\|\mathfrak{A}^{I}\right\|\left\|f^{I}(\hat{x}(t), \hat{y}(t))\right\|+\left\|\mathfrak{A}^{R}\right\|\left\|f^{R}(x(t), y(t))\right\|+\left\|\mathfrak{A}^{I}\right\|\left\|f^{R}(x(t), y(t))\right\|+\left\|\mathfrak{A}_{1}^{I}\right\|\left\|f_{1}^{I}(x(t), y(t))\right\| \\
& +\left\|\mathfrak{B}^{R}\right\|\left\|g^{R}(\hat{x}(t-\tau), \hat{y}(t-\tau))\right\|+\left\|\mathfrak{B}_{1}^{R}\right\|\left\|g_{1}^{R}(\hat{x}(t-\tau), \hat{y}(t-\tau))\right\| \\
& +\left\|\mathfrak{B}^{I}\right\|\left\|g^{I}(\hat{x}(t-\tau), \hat{y}(t-\tau))+\right\| \mathfrak{B}_{1}^{I}\|\| g_{1}^{I}(\hat{x}(t-\tau), \hat{y}(t-\tau))+\left\|I_{1}^{R}-\hat{I}_{1}^{R}\right\| \\
& \left.+\omega_{1}^{R}\left\|e^{R}(t)\right\|+\xi_{1}^{R}\left\|e^{\alpha_{1}^{R}}(t)\right\|-\rho^{R}(t)\right]-\omega_{2} S^{R^{T}}(t) S(t)-\xi_{2}^{R} \sum_{i=1}^{n} S_{i}^{\left(\alpha_{2}+1\right)}(t) \\
& +\left\|S^{I}(t)\right\|_{1}\left[\|\mathfrak{D}\|\left\|e^{I}(t)\right\|+\left\|\mathfrak{A}^{R}\right\|\left\|\phi^{I}\left(e^{R}(t), e^{I}(t)\right)\right\|\right. \\
& +\left\|\mathfrak{A}^{I}\right\|\left\|\phi^{R}\left(e^{R}(t), e^{I}(t)\right)\right\|+\left\|\mathfrak{B}^{R}\right\|\left\|\xi^{I}\left(e^{R}(t-\tau), e^{I}(t-\tau)\right)\right\|+\left\|\mathfrak{B}^{I}\right\|\left\|\xi^{R}\left(e^{R}(t-\tau), e^{I}(t-\tau)\right)\right\| \\
& +\left\|\mathfrak{D}^{*}\right\|\left\|\hat{y}^{I}(t)\right\|+\left\|\mathfrak{A}^{R}\right\|\left\|f^{I}\left(\hat{x}^{R}(t), \hat{y}^{I}(t)\right)\right\|+\left\|\mathfrak{A}_{1}^{R}\right\|\left\|f_{1}^{I}\left(\hat{x}^{R}(t), \hat{y}^{I}(t)\right)\right\| \\
& +\left\|\mathfrak{A}^{I}\right\|\left\|f^{R}\left(\hat{x}^{R}(t), \hat{y}^{I}(t)\right)\right\|+\left\|\mathfrak{A}_{1}^{I}\right\|\left\|f_{1}^{R}\left(\hat{x}^{R}(t), \hat{y}^{I}(t)\right)\right\|+\left\|\mathfrak{B}^{R}\right\|\left\|g^{I}\left(\hat{x}^{R}(t-\tau), \hat{y}^{I}(t-\tau)\right)\right\| \\
& +\left\|\mathfrak{B}_{1}^{R}\right\|\left\|g_{1}^{I}\left(\hat{x}^{R}(t-\tau), \hat{y}^{I}(t-\tau)\right)\right\|+\left\|\mathfrak{B}^{I}\right\|\left\|g^{R}\left(\hat{x}^{R}(t-\tau), \hat{y}^{I}(t-\tau)\right)\right\| \\
& \left.+\left\|\mathfrak{B}_{1}^{I}\right\|\left\|g_{1}^{R}\left(\hat{x}^{R}(t-\tau), \hat{y}^{I}(t-\tau)\right)\right\|+\left\|I^{I}-\hat{I}_{1}^{I}\right\|+\omega_{1}^{I}\left\|e^{I}(t)\right\|+\xi_{1}^{I}\left\|e^{\alpha_{1}^{I}}(t)\right\|-\rho^{I}(t)\right] \\
& -\omega_{2} S^{I^{T}}(t) S(t)-\xi_{2}^{I} \sum_{i=1}^{n} S_{i}^{\left(\alpha_{2}+1\right)^{I}}(t) \\
& \leqslant\left\|S^{R}(t)\right\|_{1}\left[\left[\|\mathfrak{D}\|+\left(\left\|\mathfrak{A}^{R}\right\|+\left\|\mathfrak{B}^{R}\right\|\right) \delta^{R R}+\left(\left\|\mathfrak{A}^{I}\right\|+\left\|\mathfrak{B}^{I}\right\|\right) \delta^{I R}+\omega_{1}^{R}\right] \zeta_{2}^{R}+\left[\left(\left\|\mathfrak{A}^{R}\right\|+\left\|\mathfrak{B}^{R}\right\|\right) \delta^{R I}\right.\right. \\
& \left.+\left(\left\|\mathfrak{A}^{I}\right\|+\left\|\mathfrak{B}^{I}\right\|\right) \delta^{I I}\right] \zeta_{2}^{I}+\left[\left\|\mathfrak{D}^{*}\right\|+\left(\left\|\mathfrak{A}^{R}\right\|+\left\|\mathfrak{A}_{1}^{R}\right\|\right)\left\|\mathcal{L}_{1}^{R}\right\|\right]\left\|\hat{x}^{R}(t)\right\|+\left[\left\|\mathfrak{A}^{R}\right\|\left\|\mathcal{L}_{1}^{R}\right\|\right. \\
& \left.+\left\|\mathfrak{A}_{1}^{I}\right\|\left\|\mathcal{L}_{1}^{I}\right\|\right]\left\|\hat{y}^{I}(t)\right\|+\left[\left\|\mathfrak{B}^{R}\right\|\left\|\mathcal{L}_{2}^{R}\right\|+\left\|\mathfrak{B}_{1}^{I}\right\|\left\|\mathcal{L}_{2}^{I}\right\|\right]\left\|\hat{x}^{R}(t-\tau)\right\|+\left[\left\|\mathfrak{B}^{I}\right\|\left\|\mathcal{L}_{2}^{I}\right\|\right. \\
& \left.\left.+\left\|\mathfrak{B}_{1}^{I}\right\|\left\|\mathcal{L}_{2}^{I}\right\|\right]\|\hat{y}(t-\tau)\|+\left(\zeta_{1} \varepsilon_{1}^{\alpha_{1}}\right)^{R}+\left\|I_{1}^{R}-\hat{I}_{1}^{R}\right\|-\rho^{R}(t)\right]-\omega_{2} S^{R^{T}}(t) S^{R}(t)-\xi_{2}^{R} \sum_{i=1}^{n} S_{i}^{\left(\alpha_{2}+1\right)^{R}}(t) \\
& +\left\|S^{I}(t)\right\|_{1}\left[\left[\|\mathfrak{D}\|+\left(\left\|\mathfrak{A}^{R}\right\|+\left\|\mathfrak{B}^{R}\right\|\right) \delta^{R I}+\left(\left\|\mathfrak{A}^{I}\right\|+\left\|\mathfrak{B}^{I}\right\|\right) \delta^{R R}+\omega_{2}^{R}\right] \zeta_{2}^{R}+\left[\left(\left\|\mathfrak{A}^{R}\right\|+\left\|\mathfrak{B}^{R}\right\|\right) \delta^{I I}\right.\right. \\
& \left.+\left(\left\|\mathfrak{A}^{I}\right\|+\left\|\mathfrak{B}^{I}\right\|\right) \delta^{I R}+\omega_{2}^{I}\right] \zeta_{2}^{I}+\left[\left\|\mathfrak{D}^{*}\right\|+\left(\left\|\mathfrak{A}^{R}\right\|+\left\|\mathfrak{A}_{1}^{R}\right\|\right)\left\|\mathcal{L}_{1}^{I}\right\|\right]\left\|\hat{y}^{I}(t)\right\|+\left[\left\|\mathfrak{A}^{I}\right\|\left\|\mathcal{L}_{1}^{R}\right\|\right. \\
& \left.+\left\|\mathfrak{A}_{1}^{I}\right\|\left\|\mathcal{L}_{1}^{R}\right\|\right]\left\|\hat{x}^{R}(t)\right\|+\left[\left\|\mathfrak{B}^{R}\right\|\left\|\mathcal{L}_{2}^{I}\right\|+\left\|\mathfrak{B}_{1}^{R}\right\|\left\|\mathcal{L}_{2}^{I}\right\|\right]\left\|\hat{x}^{R}(t-\tau)\right\|+\left[\left\|\mathfrak{B}^{I}\right\|\left\|\mathcal{L}_{2}^{R}\right\|\right. \\
& \left.\left.+\left\|\mathfrak{B}_{1}^{I}\right\|\left\|\mathcal{L}_{2}^{R}\right\|\right]\left\|\hat{y}^{I}(t-\tau)\right\|-\rho^{I}(t)\right]-\omega_{2} S^{I^{T}}(t) S^{I}(t)-\xi_{2}^{I} \sum_{i=1}^{n} S_{i}^{\left(\alpha_{2}+1\right)^{I}}(t), \\
& \leqslant-\omega_{2} S^{R^{T}}(t) S^{R}(t)-\xi_{2}^{R} \sum_{i=1}^{n} S_{i}^{\left(\alpha_{2}+1\right) R}(t)-\omega_{2} S^{I^{T}}(t) S^{I}(t)-\xi_{2}^{I} \sum_{i=1}^{n} S_{i}^{\left(\alpha_{2}+1\right) I}(t), \\
& =-2 \omega_{2}\left(S^{R^{T}}(t) S^{R}(t)+S^{I^{T}}(t) S^{I}(t)\right)-\xi_{2} 2^{\left(\alpha_{2}+1\right) / 2}\left(V^{\left(\alpha_{2}+1\right) / 2}(t)\right), \\
& \leqslant-2 \omega_{2} V(t)-\xi_{2} 2^{\left(\alpha_{2}+1\right) / 2}\left(V^{\left(\alpha_{2}+1\right) / 2}(t)\right) \text {. }
\end{aligned}
$$

When $t=0$ we have,

$$
\dot{V}(t) \leqslant-2 \omega_{2} V(t)-\xi_{2} 2^{\left(\alpha_{2}+1\right) / 2}\left(V^{\left(\alpha_{2}+1\right) / 2}(t)\right)
$$

Mathematical Modeling and Computing, Vol. 8, No. 2, pp. 228-240 (2021) 
From Lemma 1, (14) and (15) there exists $t_{s}$ such that $t_{s} \leqslant \frac{\varpi}{\omega_{2}\left(1-\alpha_{2}\right)}$ where, $\varphi=\ln \left(\omega_{2} V^{1-\alpha_{2}}(0)+\right.$ $\left.\zeta_{2} 2^{\left(\alpha_{2}+1\right) / 2}\right) / \zeta_{2} 2^{\left(\alpha_{2}+1\right) / 2}$ such that $V(t)=0$. This ensures that the synchronization errors $(7)$ and $(8)$ must converge to zero in a finite time $t_{s}+t_{e}$ for any given finite time $t$. Thus, the finite-time synchronization between time-invariant uncertainty neural networks (5) and (6) is ensured by the predefined SMS and the corresponding designed SMC. This completes the proof of theorem.

\section{Numerical example}

In this section, one numerical example is presented to illustrate the effectiveness of the theoretical results derived in the previous section.

Consider the following parameters for time invariant uncertainty neural networks (5) and (6) $x^{R}(t)=\left(x_{1}^{R}(t), x_{2}^{R}(t)\right)^{T}, x^{I}(t)=\left(x_{1}^{I}(t), x_{2}^{I}(t)\right)^{T}, f_{1}^{R}(\cdot)=f_{1}^{I}(\cdot)=f^{R}(\cdot)=f^{I}(\cdot)=g_{1}^{R}()=.g_{1}^{I}(\cdot)=$ $g^{R}(\cdot)=g^{I}(\cdot)=\tanh (\cdot)$

$$
\begin{gathered}
\bar{A}^{R}=\left(\begin{array}{cc}
2 & -1.2 \\
1.8 & 1.71
\end{array}\right), \bar{A}_{1}^{R}=\left(\begin{array}{cc}
2 & -1.2 \\
1.8 & 1.71
\end{array}\right), \bar{A}^{I}=\left(\begin{array}{cc}
1.9 & -1.25 \\
1.83 & 1.7
\end{array}\right), \bar{A}_{1}^{I}=\left(\begin{array}{cc}
1.9 & -1.25 \\
1.83 & 1.7
\end{array}\right), \\
\bar{B}^{R}=\left(\begin{array}{cc}
1.5 & 0 \\
1.3 & 1.5
\end{array}\right), \bar{B}_{1}^{R}=\left(\begin{array}{cc}
1.5 & 0 \\
1.3 & 1.5
\end{array}\right), \bar{B}^{I}=\left(\begin{array}{cc}
1.4 & 0.1 \\
1.2 & 1.6
\end{array}\right), \bar{B}_{1}^{I}=\left(\begin{array}{cc}
1.4 & 0.1 \\
1.2 & 1.6
\end{array}\right) \\
\bar{D}=\operatorname{diag}(5,5), \quad \Delta D(t)=\operatorname{diag}(0.02 \sin (t-0.1),-0.1 \sin (t-0.1)) \\
\Delta A^{R}(t)=\operatorname{diag}(0.01 \sin (t-0.1),-0.1 \sin (t-0.1)) \\
\Delta A^{I}(t)=\operatorname{diag}(0.01 \sin (t-0.1),-0.1 \sin (t-0.1)) \\
\Delta A_{1}^{R}(t)=\operatorname{diag}(0.011 \sin (t-0.1),-0.1 \sin (t-0.12)) \\
\Delta A_{1}^{I}(t)=\operatorname{diag}(0.011 \sin (t-0.1),-0.1 \sin (t-0.12)) \\
\Delta B^{R}(t)=\operatorname{diag}(0.02 \sin (t-0.1),-0.1 \sin (t-0.12)) \\
\Delta B^{I}(t)=\operatorname{diag}(0.02 \sin (t-0.1),-0.1 \sin (t-0.12)) \\
\Delta B_{1}^{R}(t)=\operatorname{diag}(0.01 \sin (t-0.1),-0.1 \sin (t-0.2)) \\
\Delta B_{1}^{I}(t)=\operatorname{diag}(0.01 \sin (t-0.1),-0.1 \sin (t-0.2))
\end{gathered}
$$

From Figs. 1-3, the finite-time synchronization behavior of the neural networks (5) and (6) is given with different time delays (i), time varying delays $\tau(t)=1.7 t, 0.3 e^{t}$ and (ii) constant delay $\tau=0.6$. This shows the correctness of the theoretical results.

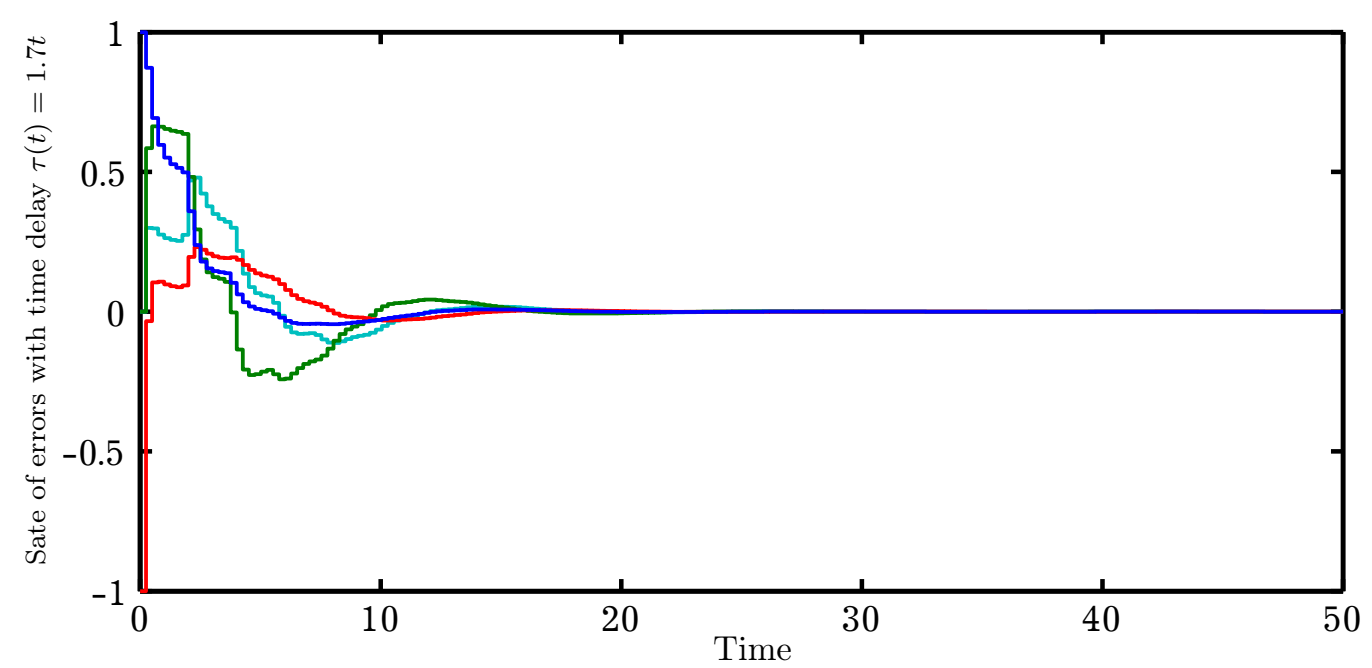

Fig. 1. Error vectors of (5) and (6) with given parameters in the numerical example section. 

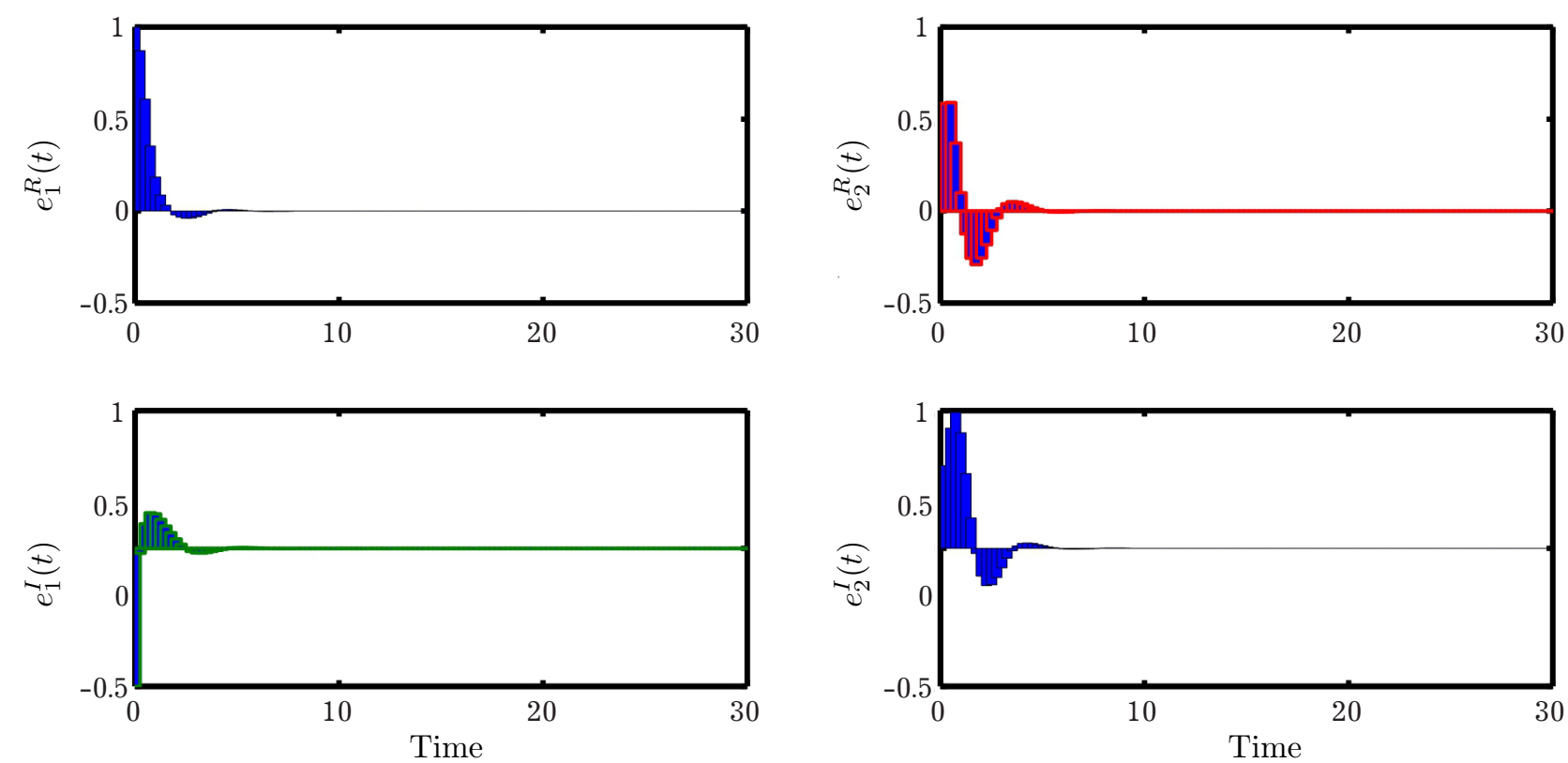

Fig. 2. Error vectors of (5) and (6) with $\tau(t)=0.3 e^{t}$ for given parameters in the numerical example section.

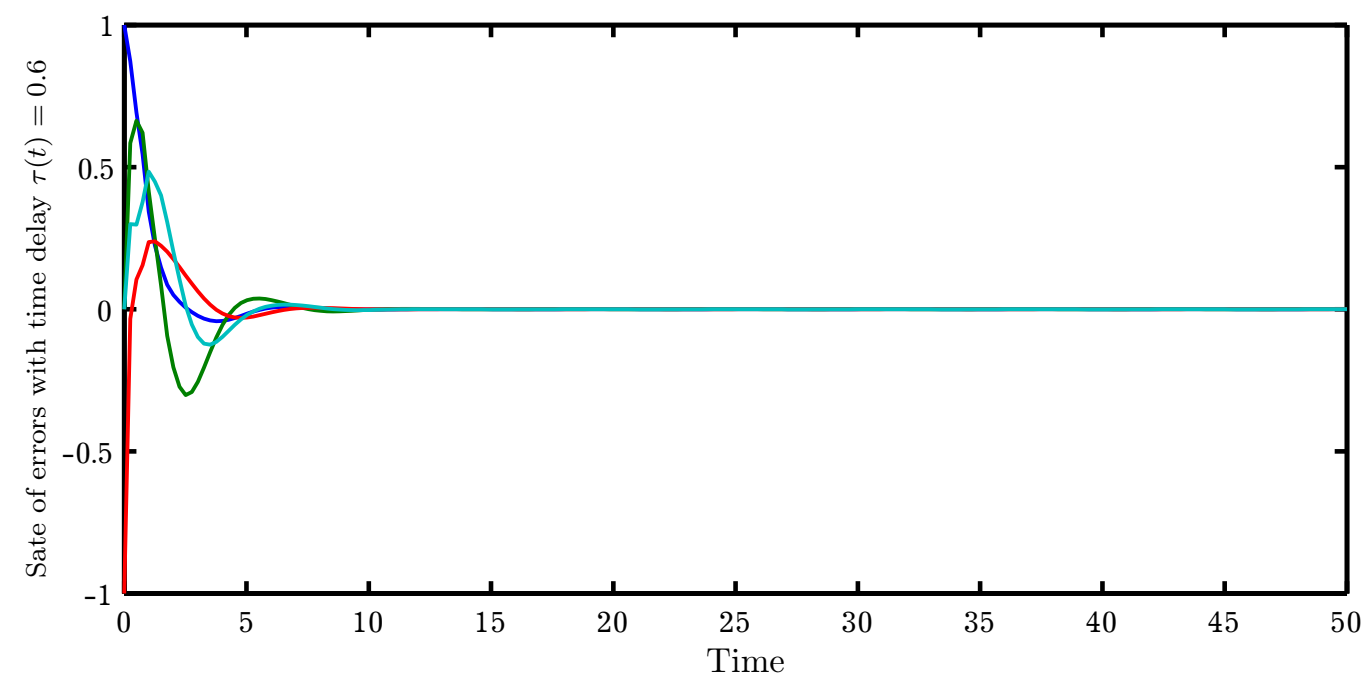

Fig. 3. Error vectors of (5) and (6) with given parameters in the numerical example section.

Remark 4. In [28], to address the problem of finite time synchronization of nonidentical delayed recurrent neural network, an improved sliding mode control approach is presented by means of drive response concept and suitable sliding mode controller is designed for the synchronization error system of real valued delayed recurrent neural networks. But in this article, we consider the complex valued neural networks with time variant uncertainty connection weight matrices and the generalized neural network structure of (1) in [28] and introduced the time variant matrices, when choosing the time variant matrices as a constant one. Therefore, the above findings are extremely generalize the existing works on the finite time synchronization of delayed recurrent neural networks.

\section{Conclusions}

In this paper, we have derived the finite-time synchronization problem of delayed complex valued neural networks with time-invariant uncertainty through improved integral sliding mode control. Firstly, the 
master-slave complex valued neural networks are transformed into two real valued neural networks. Meanwhile, the interval uncertainty terms of delayed complex valued neural networks are converted into the real uncertainty terms. Secondly, a new integral sliding mode surface is designed by employing the master-slave concept and the synchronization error of master-slave systems such that the error system can converge to zero in finite-time along the constructed integral SMS. Next, a suitable SMC is designed by using Lyapunov stability theory such that state trajectories of the system can be driven onto the predefined SMS in finite time. Finally, a numerical example is given to find the effectiveness of the theoretical results.

[1] You X., Song Q., Zhao Z. Existence and finite-time stability of discrete fractional-order complex-valued neural networks with time delays. Neural Networks. 123, 248-260 (2020).

[2] Zhang T., Han S., Zhou J. Dynamic behaviours for semi-discrete stochastic Cohen-Grossberg neural networks with time delays. Journal of the Franklin Institute. 357 (17), 13006-13040 (2020).

[3] Zhang Z., Guo R., Liu X., Zhong M., Lin C., Chen B. Fixed-time synchronization for complex-valued BAM neural networks with time delays. Asian Journal of Control. 23 (1), 298-314 (2021).

[4] Liu P., Li L., Shi K,. Lu J. Pinning stabilization of probabilistic boolean networks with time delays. IEEE Access. 8, 154050-154059 (2020).

[5] Wei R., Cao J. Global exponential synchronization of quaternion-valued memristive neural networks with time delays. Nonlinear Analysis: Modelling and Control. 25 (1), 36-56 (2020).

[6] Vadivel R., Hammachukiattikul P., Rajchakit G., Ali M. S., Unyong B. Finite-time event-triggered approach for recurrent neural networks with leakage term and its application. Mathematics and Computers in Simulation. 182, 765-790 (2021).

[7] Syed Ali M., Hymavathi M. Synchronization of Fractional Order Neutral Type Fuzzy Cellular Neural Networks with Discrete and Distributed Delays via State Feedback Control. Neural Processing Letters. 53, 929-957 (2021).

[8] Zhang Z., Guo R., Liu X., Zhong M., Lin C., Chen B. Fixed-time synchronization for complex-valued BAM neural networks with time delays. Asian J. Control. 23, 298-314 (2021).

[9] Yang S., Hu C., Yu J., Jiang H. Finite-time cluster synchronization in complex-variable networks with fractional-order and nonlinear coupling. Neural Networks. 135, 212-224 (2021).

[10] Duan L., Shi M., Huang C., Fang X. Synchronization in finite-/fixed-time of delayed diffusive complexvalued neural networks with discontinuous activations. Chaos, Solitons \& Fractals. 142, 110386 (2021).

[11] Hu T., He Z., Zhang X., Zhong S. Finite-time stability for fractional-order complex-valued neural networks with time delay. Applied Mathematics and Computation. 365, 124715 (2020).

[12] Wang Z., Liu X. Exponential stability of impulsive complex-valued neural networks with time delay. Mathematics and Computers in Simulation. 156, 143-157 (2019).

[13] Chanthorn P., Rajchakit G., Thipcha J., Emharuethai C., Sriraman R., Lim C. P., Ramachandran R. Robust stability of complex-valued stochastic neural networks with time-varying delays and parameter uncertainties. Mathematics. 8 (5), 742 (2020).

[14] Syed Ali M., Narayanan G., Shekher V., Alsaedi A., Ahmad B. Global Mittag-Leffler stability analysis of impulsive fractional-order complex-valued BAM neural networks with time varying delays. Communications in Nonlinear Science and Numerical Simulation. 83, 105088 (2020).

[15] Li H.-L., Hu C., Cao J., Jiang H., Alsaedi A. Quasi-projective and complete synchronization of fractionalorder complex-valued neural networks with time delays. Neural Networks. 118, 102-109 (2019).

[16] Zhang W., Zhang H., Cao J., Alsaadi F. E., Chen D. Synchronization in uncertain fractional-order memristive complex-valued neural networks with multiple time delays. Neural Networks. 110, 186-198 (2019).

[17] Panteley E., Loría A. Synchronization and dynamic consensus of heterogeneous networked systems. IEEE Trans. Automat. Contr. 62 (9), 3758-3773 (2017).

[18] Wang X. F., Chen G. R. Synchronization in scale-free dynamical networks: robustness and fragility. IEEE Transactions on Circuits and Systems I: Fundamental Theory and Application. 49 (1), 54-62 (2002).

Mathematical Modeling and Computing, Vol. 8, No. 2, pp. 228-240 (2021) 
[19] Sun Y. Z., Li W., Ruan J. Generalized outer synchronization between complex dynamical networks with time delay and noise perturbation. Communications in Nonlinear Science and Numerical Simulation. 18 (4), 989-998 (2013).

[20] Li C. G., Chen G. R. Phase synchronization in small-world networks of chaotic oscillators. Physica A: Statistical Mechanics and its Applications. 341, 73-79 (2004).

[21] Cao J., Ho D. W. C., Yang Y. Q. Projective synchronization of a class of delayed chaotic systems via impulsive control. Physics Letters A. 373, 3128-3133 (2009).

[22] Li C. D., Liao X. F., Wong K. W. Chaotic lag synchronization of coupled time-delayed systems and its applications in secure communication. Physica D: Nonlinear Phenomena. 194 (3-4), 187-202 (2004).

[23] Zhou J., Lu J. A., Lü J. Adaptive synchronization of an uncertain complex dynamical network. IEEE Transactions on Automatic Control. 51 (4), 1339-1344 (2006).

[24] Zhou J., Lu J. A., Lu J. Pinning adaptive synchronization of a general complex dynamical network. Automatica. 44 (4), 996-1003 (2008).

[25] Zhao Y., Li X., Duan P. Observer-based sliding mode control for synchronization of delayed chaotic neural networks with unknown disturbance. Neural Networks. 117, 268-273 (2019).

[26] Huang H., Feng G. Synchronization of nonidentical chaotic neural networks with time delays. Neural Networks. 22 (7), 869-874 (2009).

[27] Zhang D., Xu J. Projective synchronization of different chaotic time-delayed neural networks based on integral sliding mode controller. Applied Mathematics and Computation. 217 (1), 164-174 (2010).

[28] Xiong J. J., Zhang G. B., Wang J. X., Yan T. H. Improved sliding mode control for finite-time synchronization of nonidentical delayed recurrent neural networks. IEEE Transactions on Neural Networks and Learning Systems. 31 (6), 2209-2216 (2019).

\title{
Синхронізація інваріантних щодо часу невизначених нейронних мереж із затримкою на скінченний час за рахунок вдосконаленого керування режимом ковзання
}

\author{
Джаянті H. ${ }^{1}$, Сантакумарі P. ${ }^{1,2}$ \\ ${ }^{1}$ Урлдовий коледж мистецтв, Коӥмбаторі, Індія \\ ${ }^{2}$ Коледж мистецтв і науки Шрі Рамакрішна, Коїмбаторі, Індія
}

\begin{abstract}
У статті досліджується задача часово-скінченної синхронізації складних нейронних мереж із запізнюванням та інваріантною щодо часу невизначеністю шляхом вдосконалення інтегрального керування режимом ковзання. По-перше, комплексні нейронні мережі "ведучий-ведений" перетворюються на дві дійсні нейронні мережі за допомогою методу поділу комплексних нейронних мереж на дійсну та уявну частини. Крім того, члени інтервальної невизначеності комплексних нейронних мереж із запізнюванням перетворюються на дійсні умови невизначеності. По-друге, нова інтегральна поверхня ковзного режиму розроблена з використанням концепції "ведучий-ведений" так, що система помилок може збігатися до нуля за скінченний час вздовж побудованої інтегральної поверхні режиму ковзання. Далі, за допомогою теорії стійкості Ляпунова розроблено відповідне керування режимом ковзання, завдяки якому траєкторії стану системи можуть бути переведені на попередньо задану поверхню режиму ковзання за скінченний час. Нарешті, подано чисельний приклад, який ілюструє ефективність теоретичних результатів.
\end{abstract}

Ключові слова: керування режсимм ковзання, поверхня режиму ковзання, часова невизначеність, часова затримка, нейронні мережі.

Mathematical Modeling and Computing, Vol. 8, No. 2, pp. 228-240 (2021) 